\title{
Aushangpflicht nicht beachtet: Das kann teuer werden!
}

— Arbeitgeber - und dazu zählen natürlich auch niedergelassene Ärzte - sind verpflichtet, ihre Arbeitnehmer über arbeitsschutzrelevante Gesetze zu informieren. Je nach Regelung soll dies in geeigneter Weise durch Auslegen, Aushängen oder Bekanntmachung geschehen. Eine Pflicht zum Aushang oder zur Auslage entsteht insbesondere dann, wenn in der Arbeitsstätte Gesetze, Verordnungen oder Vorschriften anzuwenden sind, die Aushangsoder Auslagebestimmungen enthalten.

Manche Aushangs-/Auslagepflichten sind an die Beschäftigungszahl geknüpft. So sind das Arbeitszeitgesetz und die Unfallverhütungsvorschriften (je nach Branche) bereits bei regelmäßig beschäftigten Arbeitnehmern auszuhängen bzw. bekannt zu geben. Das Jugendarbeitsschutzgesetz ist bei Beschäftigung mindestens eines Jugendlichen und das Mutterschutzgesetz bei einer regelmäßigen Beschäftigung von mehr als drei Frauen bekannt zu geben. In jedem Fall muss für den Mitarbeiter die Möglichkeit bestehen, ohne Schwierigkeiten den jeweiligen Inhalt zu erfahren. Üblicherweise erfolgt ein Aushang an einem sog. Schwarzen Brett an einer allgemein zugänglichen Stelle des Betriebes.

\section{Kommentar}

Ein Verstoß gegen die Aushangpflicht kann mit einer Geldbuße von bis zu 2500 Euro geahndet werden. Hinzu kommen i. d. R. noch die Kosten eines Verfahrens, die der Arbeitgeber zu tragen hat, wenn eine Beanstandung stattgefunden hat.

\section{Verjährungsfrist bei Regressen}

— Bei Arzneimittelregressen gilt eine Verjährungsfrist von zwei Jahren nach Abschluss des betreffenden Quartals. Diese Frist wird erst mit der Zustellung eines Prüfbescheides unterbrochen. Nach Urteilen des Bundessozialgerichts (BSG) sind Ausnahmen nur zulässig, wenn ein Prüfungsausschuss (PA) plausible Gründe für eine Verzögerung der Bescheiderteilung darlegen kann. In einem solchen Fall waren die Regressbescheide des PA mehr als vier Jahre nach Abschluss der jeweils betroffenen Quartale ergangen. Die vierjährige Ausschlussfrist war dem Gericht zufolge hier jedoch durch die Mitteilung aufgehoben, dass eine Überprüfung der Verordnungsweise der Praxis nach Durchschnittswerten erfolgen sollte, diese Prüfung aber bis zur Entscheidung über die Durchfüh- rung von Richtgrößenprüfungen zurückgestellt werden musste. In dieser Situation war der PA an einem Tätigwerden gehindert, weil nach dem Gesetz vorrangig eine Richtgrößenprüfung durchzuführen war (Az.: B 6 KA 27/11 R).

\section{Kommentar}

Da der Vertragsarzt bei Prüfungen dieser Art nur mittelbar über seine KV in die Verhandlungen einbezogen ist, fordern die Richter deshalb aber auch, dass ihm der Grund für das Zurückstellen einer Prüfung deutlich mitgeteilt wird. Wenn ein Prüfungsausschuss eine Praxis deshalb nur darüber informiert, dass eine Prüfung beabsichtigt ist, reiche dies zur Unterbrechung der Verjährungsfrist nicht aus (Az.: B 6 KA 45/11 R). 